\title{
Hematological Indices May Be Useful in the Diagnosis of Systemic Lupus Erythematosus and in Determining Disease Activity in Behçet's Disease
}

\author{
Servet Yolbas ${ }^{\mathrm{a}} \quad$ Ahmet Yildirim $^{\mathrm{a}}$ Nevzat Gozel ${ }^{\mathrm{b}}$ Burak Uz \\ Suleyman Serdar Koca ${ }^{a}$ \\ Departments of ${ }^{a}$ Rheumatology, ${ }^{b}$ Internal Medicine and ${ }^{\mathrm{c} H e m a t o l o g y, ~ F a c u l t y ~ o f ~ M e d i c i n e, ~ F i r a t ~ U n i v e r s i t y, ~}$ \\ Elazig, Turkey
}

\section{Key Words}

Rheumatic diseases · Systemic lupus erythematosus .

Behçet's disease $\cdot$ Hematological indices

\begin{abstract}
Objectives: The aim of this study was to investigate the relationships between clinical features of rheumatic diseases and hematologic indices, including mean platelet volume (MPV), MPV/platelet ratio (MPR), platelet/lymphocyte ratio, and neutrophil/lymphocyte ratio (NLR). Subjects and Methods: Rheumatoid arthritis (RA; $n=91)$, systemic lupus erythematosus (SLE; $n=51$ ), systemic sclerosis (SSc; $n=39$ ), and Behçet's disease (BD; $n=53$ ) patients, and 55 healthy controls $(\mathrm{HC})$ were enrolled. Hematological indices were calculated and one-way analysis of variance, Mann-Whitney $U$ and $X^{2}$ tests, and receiver operating characteristic (ROC) analyses were performed. Results: The MPV and MPR were higher in the SLE group than the RA group $(p<0.05$ and $p<0.01$, respectively). ROC analysis indicated that MPV (area under the curve, AUC, $0.68,95 \% \mathrm{Cl} 0.58-0.77$ ) and MPR (AUC 0.69, 95\% $\mathrm{Cl}$ 0.59-0.78) were sensitive and specific markers for SLE against RA. The NLR was higher in the RA, SLE, and SSc groups compared to the HC group $(p<0.05, p<0.001$, and $p<0.01$, respectively). The NLR was higher in the active $B D$ patients than those that were inactive $(p=0.008)$. Besides, NLR was
\end{abstract}

higher in patients with neuro-BD and patients with active genital ulcers compared to patients without neurological involvement $(p<0.01)$ and active genital ulcers $(p<0.05)$. Conclusion: The MPV and MPR were significantly higher in the SLE group than in the RA group. They were also higher in the active than in the inactive BD patients. The MPV and MPR are useful diagnostic tools for SLE, and NLR reflects disease activity in BD. However, further research should be performed to standardize these tools.

(c) 2016 S. Karger AG, Basel

\section{Introduction}

Abnormal immune regulation and persistent inflammation, the characteristic features of chronic inflammatory diseases, have adverse effects on the hematopoietic system. Changes in peripheral blood cell components are used to detect disease activity and to diagnose some collagen tissue diseases such as rheumatoid arthritis (RA) [1-3], systemic lupus erythematosus (SLE) [4, 5] and systemic sclerosis (SSc) [6]. Rheumatic inflammatory diseases affect one or more cellular lineages of the hematologic system, and thus frequent hematologic manifestations, such as anemia, neutropenia, thrombocytopenia, and hematologic malignancies, are observed in these dis-

\begin{tabular}{ll}
\hline KARGER & $\begin{array}{l}\text { ○ 2016 S. Karger AG, Basel } \\
1011-7571 / 16 / 0256-0510 \$ 39.50 / 0 \quad \text { Karger }\end{array}$ \\
E-Mail karger@karger.com & $\begin{array}{l}\text { Thisis an Open Access article licensed under the terms of the } \\
\text { Creative Commons Attribution-NonCommercial 3.0 Un- } \\
\text { ported license (CC BY-NC) (www.karger.com/OA-license), } \\
\text { applicable to the online version of the article only. Distribu- } \\
\text { tion permitted for non-commercial purposes only. }\end{array}$
\end{tabular}

Prof. Suleyman Serdar Koca, MD

Firat Universitesi Hastanesi Romatoloji Klinigi

TR-23200 Elazig (Turkey)

E-Mail kocassk@yahoo.com 
eases. These hematologic abnormalities are due to several immune- and nonimmune-mediated mechanisms. The causes of hematological abnormalities are excessive production of cytokines, antibodies, immune complexes, growth factor deficiencies, increased peripheral accumulation, a decreased life span, decreased neutrophil functions, gastrointestinal losses, and medication-related toxicities [1-6].

RA [1-3], SLE [4, 5], and other inflammatory diseases $[1,3,6-9]$ affect the numbers, ratios, and volumes of peripheral blood cells. Mean platelet volume (MPV) is a parameter of a complete blood cell count (CBC). It reflects the function and activation of platelets, and has been studied in many inflammatory, cardiovascular and cerebrovascular diseases $[1,4,6,8,10]$. Large platelets include more granules than small platelets. These granules release prothrombotic factors, such as thromboxane A2, serotonin, $\beta$-thromboglobulin, and adenosine triphosphate, and affect inflammatory and endothelial functions. They also include the expression of adhesion factors, such as $\mathrm{P}$-selectin and glycoprotein IIb/IIIa, and increase vasoconstriction [10].

Recent studies have shown the numbers and ratios of white blood cell subgroups in inflammatory rheumatic diseases $[3,4,9]$. However, the relationship between hematologic indices and rheumatologic diseases are still controversial [1, 2, 4, 5, 10-12]. Therefore, we aimed to investigate the possible relationships of various indices, such as MPV, MPV/platelet ratio (MPR), platelet/lymphocyte ratio (PLR), and neutrophil/lymphocyte ratio (NLR), on disease activities and clinical processes of several rheumatic inflammatory diseases.

\section{Subjects and Methods}

\section{Patient Selection}

Ninety-one patients with RA, 51 patients with SLE, 39 patients with SSc, 53 patients with Behçet's disease (BD) and 55 healthy volunteers (healthy controls; HC) were included in this study. The patients met established criteria used for diagnosis and classification in routine practice [13-16].

The exclusion criteria of the study were participants younger than 18 years of age, older than 80 years, evidence of infection, pregnancy, receiving antiplatelet medications such as aspirin and clopidogrel, atherosclerotic disease, diabetes mellitus, hypertension, and dyslipidemia. The protocol of this study was approved by the institutional ethics committee, and informed consent was obtained from all the participants before they enrolled in the study. Detailed histories of all the participants were obtained from medical records and systemic and rheumatologic examinations were performed. The clinical process of the participants and their treatment protocols were also recorded.

Hematological Indices in Rheumatic Diseases
Disease Activity and Severity Scoring

Disease activity and/or severity scores were determined by the disease activity score-28 (DAS-28) in the RA group; SLE disease activity index (SLEDAI) and Systemic Lupus International Collaborating Clinics/American College of Rheumatology (SLICC/ ACR) damage index in the SLE group. Behçet's syndrome activity score (BSAS) was employed in the BD group. The Valentini disease activity index and Medsger disease severity scale were used in the SSc group. In addition, skin involvement was detected via the modified Rodnan skin score (mRSS) in the SSc group [17-21]. Active diseases in RA, SSc and SLE were denoted by a DAS- $28 \geq 2.6$; Valentini disease activity index $\geq 3$, and SLEDAI $\geq 6$, respectively. In the $\mathrm{BD}$ group, the disease was interpreted as active when a patient had at least two of the following: genital ulcers, skin lesions, active arthritis, recent eye involvement, recent neurological involvement, recent vascular involvement, a positive pathergy test sign in addition to oral ulcer, as well as a high erythrocyte sedimentation rate (ESR) and/or high C-reactive protein (CRP) levels.

\section{Laboratory Analyses}

Blood samples were drawn from the participants after they had fasted overnight. Routine laboratory evaluations, including $\mathrm{CBC}$ and tests of ESR, CRP, creatinine, and total creatinine kinase, were performed using standard laboratory methods. In addition, autoantibodies (antinuclear antibody-IFA, anti-ds-DNA, anti-Sm, anticentromere and anti-Scl-70 antibodies) were analyzed using standard methods in the SLE and SSc groups. The blood samples for analysis of hematological parameters were collected in tubes containing ethylenediaminetetraacetic acid, and all laboratory analyses were performed on the same day within $1 \mathrm{~h}$ after the collection of samples. The MPV, MPR, PLR, and NLR ratios were calculated from the $\mathrm{CBC}$ results.

\section{Statistical Analyses}

Statistical analysis was performed using the Statistical Package for the Social Sciences 21 (SPSS, Chicago, Ill., USA). The results are presented as the mean \pm standard deviation. The normal distribution of the variables was evaluated with the KolmogorovSmirnov test, and data with a skewed distribution (ESR, CRP, MPR, PLR, and NLR) were expressed as the median (minimummaximum). Statistical differences among the groups were identified with a one-way analysis of variance (ANOVA), followed by Tukey's post hoc test for parametric data, while Kruskal-Wallis and post hoc Mann-Whitney $U$ tests were used for nonparametric data. A $\chi^{2}$ test was used to compare the categorical variables. Correlation analysis was performed using Pearson's correlation coefficient. The diagnostic efficacies of MPV and MPR for SLE were examined by receiver operating characteristic (ROC) analysis. A $p$ value $<0.05$ was considered significant.

\section{Results}

The demographics and clinical data of the study groups are summarized in table 1 . The DAS-28 ESR was $3.2 \pm 1.5$ in the RA group. The SLEDAI and SLICC/ACR were 12.1 \pm 9.5 and $1.7 \pm 1.5$, respectively, in the SLE group. The mRSS and Medsger scores were $14.5 \pm 7.3$ and $5.9 \pm 2.7$, 
Table 1. Demographic and clinical characteristics of the study groups

\begin{tabular}{|c|c|c|c|c|c|c|}
\hline & $\mathrm{RA}(\mathrm{n}=91)$ & $\operatorname{SLE}(n=51)$ & $\operatorname{SSc}(n=39)$ & $\mathrm{BD}(\mathrm{n}=53)$ & $\mathrm{HC}(\mathrm{n}=55)$ & $\mathrm{p}$ values \\
\hline Age, years & $51.7 \pm 14.5^{*}$ & $33 \pm 9.6^{* * *}$ & $49.2 \pm 13.2$ & $37.4 \pm 10.9^{*}$ & $45.1 \pm 13$ & $<0.001^{\mathrm{a}}$ \\
\hline Sex, F/M & $76 / 15$ & $47 / 4$ & $35 / 4$ & $33 / 20$ & $44 / 11$ & $0.001^{\mathrm{b}}$ \\
\hline BMI & $27.7 \pm 5.3$ & $25.1 \pm 8.1$ & $25.4 \pm 5$ & $26.3 \pm 4.5$ & $27.4 \pm 4.8$ & $0.430^{\mathrm{a}}$ \\
\hline Disease duration, years & $8.2 \pm 7.9$ & $4.6 \pm 5.2$ & $8.5 \pm 5.8$ & $6.9 \pm 5.9$ & - & $0.140^{\mathrm{a}}$ \\
\hline Smoking & $11(12.1)$ & $8(15.7)$ & $4(7.6)$ & $10(18.9)$ & $7(12.7)$ & $0.836^{\mathrm{b}}$ \\
\hline
\end{tabular}

Values are expressed as means $\pm \mathrm{SD}$ or $\mathrm{n}(\%)$. When compared to the HC group: ${ }^{*} \mathrm{p}<0.05,{ }^{* * *} \mathrm{p}<0.001 .{ }^{\mathrm{a}}$ ANOVA p values. ${ }^{\mathrm{b}} \chi^{2}$ p values. ${ }^{1}$ The dose of corticosteroid equivalent to prednisolone.

Table 2. Laboratory data of the study groups

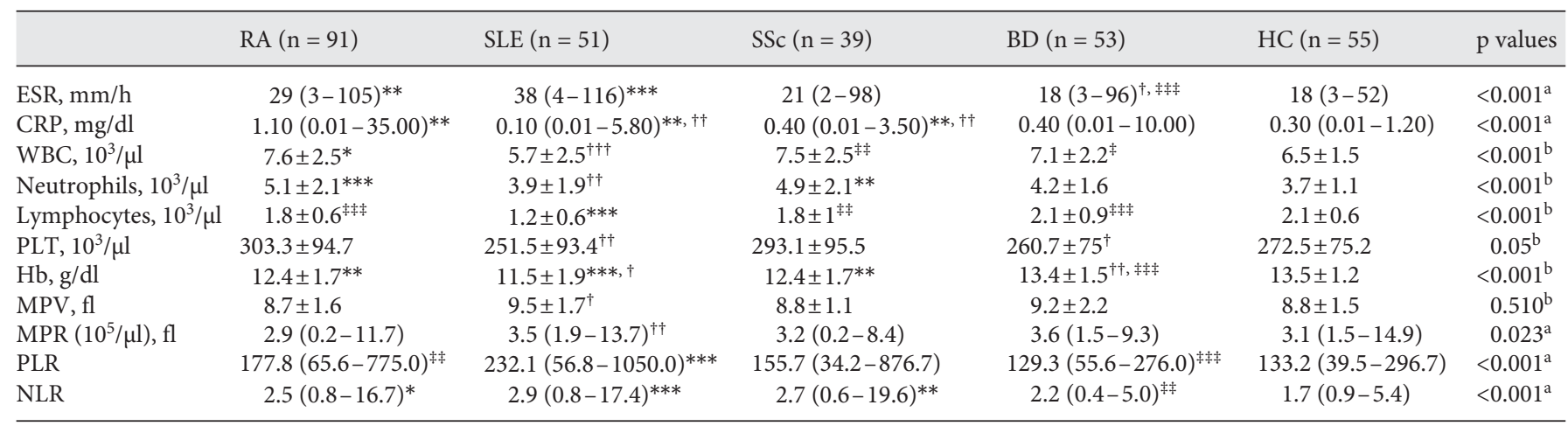

When compared to the HC group: ${ }^{*} \mathrm{p}<0.05,{ }^{* *} \mathrm{p}<0.01,{ }^{* * *} \mathrm{p}<0.001$. When compared to the RA group; ${ }^{\dagger} \mathrm{p}<0.05,{ }^{\dagger \dagger} \mathrm{p}<0.01,{ }^{\dagger \dagger \dagger} \mathrm{p}<0.001 . \mathrm{Wh}^{*} \mathrm{n}$ compared to the SLE group; ${ }^{\ddagger} \mathrm{p}<0.05,{ }^{\ddagger \ddagger} \mathrm{p}<0.01,{ }^{\ddagger \ddagger \neq} \mathrm{p}<0.001 .{ }^{\mathrm{a}}$ Kruskal-Wallis $\mathrm{p}$ values. ${ }^{\mathrm{b}}$ ANOVA $\mathrm{p}$ values. WBC $=$ White blood cell; PLT $=$ platelets; $\mathrm{Hb}=$ hemoglobin.

respectively, in the SSc group. The BSAS was $14.6 \pm 16.7$ in the BD group.

The MPV and MPR were higher in the SLE group than in the RA group ( $p<0.05$ and $p<0.01$, respectively). However, there was no statistically significant difference among the other groups in terms of the MPV and MPR (table 2). The PLR was significantly higher in the SLE group than in the HC group (table 2). However, the PLR of the RA and SSc groups were similar to that of the HC group (table 2). The NLR was significantly higher in the RA, SLE, and SSc groups than in the HC group (table 2), although it was similar in the $\mathrm{BD}$ and $\mathrm{HC}$ groups $(\mathrm{p}=$ $0.863)$.

Based on the ROC analysis, the MPV value (using a cut-off of $8.95 \mathrm{fl}$ ) differentiated SLE from RA, with a sensitivity and specificity of 70 and $62 \%$, respectively. Moreover, the MPR [using a cut-off of $3.28 \mathrm{fl} /\left(10^{5} / \mu \mathrm{l}\right)$ ] differ- entiated SLE from RA, with a sensitivity and specificity 64 and $63 \%$, respectively. These results indicated that MPV (area under the curve, AUC, $0.68,95 \%$ CI 0.58-0.77) and MPR (AUC 0.69, 95\% CI 0.59-0.78) were sensitive and specific markers for SLE.

\section{Hematologic Indices in the RA Group}

There was no significant difference in MPV, MPR, PLR, and NLR between the active $(n=52)$ and quiescent $(\mathrm{n}=39)$ RA subgroups and between the seropositive and seronegative RA subgroups ( $\mathrm{p}>0.05$ for all; data not shown). The leukocyte $\left(8.1 \pm 2.2\right.$ vs. $6.9 \pm 2.710^{3} / \mu \mathrm{l}, \mathrm{p}=$ $0.02)$ and neutrophil $\left(5.4 \pm 1.9\right.$ vs. $4.4 \pm 2.210^{3} / \mu \mathrm{l}, \mathrm{p}=$ 0.027 ) counts were significantly higher in the rheumatoid factor $(R F)$-positive $(n=56)$ than in the RF-negative cases $(n=31)$. In the RA group, the MPV and body mass index (BMI; $\mathrm{p}=0.001, \mathrm{r}=0.338)$, PLR and ESR ( $\mathrm{p}=0.011$, 
$\mathrm{r}=0.271)$, and NLR and RF titers $(\mathrm{p}=0.014, \mathrm{r}=0.263)$ were significantly correlated.

\section{Hematologic Indices in the SLE Group}

There was no significant difference in the MPV, MPR, PLR, and NLR between the active $(\mathrm{n}=37)$ and quiescent $(\mathrm{n}=17)$ SLE subgroups and between positive and negative anti-ds-DNA ( $p>0.05$ for all; data not shown). However, the hemoglobin level was significantly lower in the active subgroup compared to that of the inactive subgroup ( $10.8 \pm 1.8$ vs. $12.8 \pm 1.4 \mathrm{~g} / \mathrm{dl}, \mathrm{p}<0.001)$. It was also significantly lower in the patients with positive antids-DNA compared to negative patients $(11.1 \pm 2.0$ vs. $12.5 \pm 1.6 \mathrm{~g} / \mathrm{dl}, \mathrm{p}=0.035)$ and in the patients with proteinuria $(\mathrm{n}=15)$ compared to those without proteinuria $(\mathrm{n}=36 ; 10.1 \pm 1.5$ vs. $12.1 \pm 1.8 \mathrm{~g} / \mathrm{dl}, \mathrm{p}=0.001)$. The NLR was higher in hypocomplementemic $(n=17)$ SLE patients than in normocomplementemic patients $(n=34)$. However, there was no significant difference in the MPV, MPR, and PLR between the same subgroups ( $p>$ $0.05)$. Similarly, there was no significant difference in the MPV, MPR, PLR, and NLR between smokers $(n=8)$ and nonsmokers $(n=43)$ in the SLE group ( $p>0.05$ for all). The PLR was lower in hypertensive $(\mathrm{n}=12)$ than in normotensive $(\mathrm{n}=39)$ SLE patients $(184.7 \pm 62.9$ vs. 303.1 $\pm 246.2, \mathrm{p}=0.012$ ), but there was no statistically significant difference in the MPV, MPR, and NLR ( $p>0.05$ ). In the SLE group, 36 patients were taking a steroid, 28 were receiving azathioprine and 26 were receiving hydroxychloroquine. The MPV, MPR, PLR, and NLR of the patients receiving drug treatment were not significantly different from those not receiving a drug treatment ( $p>0.05)$.

\section{Hematologic Indices in the SSc Group}

The MPV, MPR, PLR, and NLR of the patients with active and inactive SSc, diffuse and limited subtypes, and positive and negative anticentromere or anti-Scl-70 antibodies showed no significant differences ( $p>0.05$ for all; data not shown). Similarly, there was no significant difference in the MPV, MPR, PLR, and NLR between patients with pulmonary hypertension, pulmonary fibrosis, heart, gastrointestinal system, and kidney involvement and patients who did not have these features ( $p>0.05$ for all; data not shown). In the SSc group, the Medsger severity index, Valentini activity index, and mRSS were not correlated with the MPV, MPR, PLR and NLR ( $p>0.05$ for all). The NLR was correlated with patient age $(\mathrm{r}=$ $0.349, \mathrm{p}=0.029)$ and BMI $(\mathrm{r}=0.446, \mathrm{p}=0.020)$.

Hematological Indices in Rheumatic Diseases

\section{Hematologic Indices in the BD Group}

The NLR was significantly higher $(2.7 \pm 1.1$ vs. $2 \pm 0.7$, $\mathrm{p}=0.008)$ and the MPV was significantly lower $(9 \pm 3.2$ vs. $9.3 \pm 1.3 \mathrm{fl}, \mathrm{p}=0.047)$ in the patients with active $\mathrm{BD}$ $(\mathrm{n}=19)$ compared to those with inactive BD $(\mathrm{n}=34)$. The NLR was significantly higher in the patients with neuro$\mathrm{BD}(\mathrm{n}=6)$ compared to those without $(\mathrm{n}=47 ; 3.5 \pm 1.1$ vs. $2.1 \pm 0.7, p=0.002$ ). It was also higher in the patients with active genital ulcer $(n=6)$ compared to those without $(\mathrm{n}=47 ; 3.3 \pm 1.2$ vs. $2.2 \pm 0.8, \mathrm{p}=0.012)$. However, these clinical features did not alter the levels of MPV, MPR, or PLR ( $p>0.05$ for all). There was no significant difference in the MPV, MPR, PLR, and NLR of the BD patients with $(\mathrm{n}=16)$ and without ocular involvement $(p>0.05)$. Furthermore, there was no significant difference in MPV, MPR, PLR, and NLR of the BD patients with $(\mathrm{n}=19)$ and without $(\mathrm{n}=34)$ active oral ulcers $(\mathrm{p}>0.05)$. The MPV, MPR, PLR, and NLR of the BD patients receiving steroid treatment $(\mathrm{n}=12)$, azathioprine $(\mathrm{n}=28)$, and colchicine $(\mathrm{n}=42)$ were similar to those with BD not receiving these drugs ( $p>0.05$ for all). In the BD group, there was a negative correlation between the MPV and patient age, a negative correlation between the MPR and CRP level, and positive correlations of the NLR with ESR and BSAS. In this group, there was also a negative correlation between the PLR and BMI, and a positive correlation between the PLR and ESR, and positive correlations of the MPR with age, disease duration, and ESR (table 3).

\section{Discussion}

In this study, MPV and MPR were significantly higher in the SLE group than in the RA group. PLR was also significantly higher in the SLE group than in the HC group. Furthermore, NLR was significantly higher in the RA, SLE, and SSc groups than in the HC group. NLR was significantly higher in hypocomplementemic patients than in normocomplementemic patients in the SLE group. It was also higher in the active $\mathrm{BD}$ patients than in inactive $\mathrm{BD}$ patients, and it was significantly higher in the patients with neuro-BD than in those without.

The inflammatory process in rheumatic inflammatory diseases involves inflammatory cells and molecules which cause changes in the number, shapes, and sizes of bone marrow cells and peripheral blood cells [1-12, 22, 23]. However, the effects of inflammatory diseases on hematological indices are controversial.

The levels of MPV were not higher in the RA and SSc groups, but they were slightly elevated in the SLE and BD 
Table 3. Correlation analysis of the data in the BD group

\begin{tabular}{|c|c|c|c|c|c|c|c|c|}
\hline & \multicolumn{2}{|l|}{ MPV } & \multicolumn{2}{|l|}{ MPR } & \multicolumn{2}{|l|}{ PLR } & \multicolumn{2}{|l|}{ NLR } \\
\hline & $\mathrm{r}$ & $\mathrm{p}$ & $\mathrm{r}$ & $\mathrm{p}$ & $\mathrm{r}$ & $\mathrm{p}$ & $\mathrm{r}$ & $\mathrm{p}$ \\
\hline Age & -0.146 & 0.018 & 0.180 & 0.004 & -0.003 & 0.962 & 0.030 & 0.625 \\
\hline Disease duration & -0.101 & 0.132 & 0.154 & 0.022 & -0.039 & 0.561 & -0.042 & 0.531 \\
\hline BMI & -0.051 & 0.408 & 0.010 & 0.864 & -0.157 & 0.010 & -0.074 & 0.224 \\
\hline ESR & -0.097 & 0.148 & 0.170 & 0.011 & 0.221 & 0.001 & 0.165 & 0.013 \\
\hline CRP & -0.064 & 0.356 & -0.176 & 0.011 & 0.101 & 0.148 & 0.053 & 0.445 \\
\hline WBC & -0.153 & 0.010 & 0.275 & $<0.001$ & -0.166 & 0.005 & 0.263 & $<0.001$ \\
\hline $\mathrm{Hb}$ & 0.160 & 0.007 & 0.024 & 0.686 & -0.328 & $<0.001$ & -0.151 & 0.012 \\
\hline PLT & -0.117 & 0.050 & 0.736 & $<0.001$ & 0.332 & $<0.001$ & 0.062 & 0.302 \\
\hline Neutrophil & -0.109 & 0.068 & 0.225 & $<0.001$ & 0.025 & 0.677 & 0.499 & $<0.001$ \\
\hline Lymphocyte & -0.134 & 0.024 & 0.188 & 0.002 & -0.567 & $<0.001$ & -0.498 & $<0.001$ \\
\hline BSAS & -0.057 & 0.687 & -0.017 & 0.905 & 0.154 & 0.272 & 0.369 & 0.007 \\
\hline
\end{tabular}

$\mathrm{WBC}=$ White blood cell $\mathrm{Hb}=$ hemoglobin; PLT $=$ platelets.

patients in our study. Higher levels of MPV were reported in the patients with RA [2, 22], SLE [5], SSc [6], ankylosing spondylitis (AS) [12], psoriatic arthritis [7], and $\mathrm{BD}$ [8]. On the other hand, Kisacik et al. [1] reported that levels of MPV were lower in patients with AS. Similarly, subsequent studies demonstrated the lower levels of MPV in patients with RA [1] and SLE [4]. There is a contradiction concerning whether MPV levels are increased in the inflammatory diseases. This contradiction could be explained by the differences of disease activity status of patients and their ongoing treatments. Disease activity and treatments alter MPV levels. Yazici et al. [12] reported that MPV levels decrease after treatment with biologics in patients with AS. Conversely, the MPV level was demonstrated to be increased after treatment in patients with SLE [4]. In our study, the active BD patients had lower MPV levels compared to the inactive ones. However, disease activity did not affect MPV levels in the RA, SLE and SSc groups. Moreover, our study did not show that treatments altered MPV levels.

Glucocorticoids may also affect the count, size and function of platelets. Therefore, using glucocorticoids may be another confounding factor for the studies evaluating MPV levels in inflammatory diseases. However, in our study the MPV levels were similar regardless of whether the patients received glucocorticoids or not. On the other hand, the selected anticoagulants in which blood samples are collected and times between blood sample collection and analysis can alter the MPV level [24]. Other causes to explain the contradiction regarding MPV levels between studies may also exist.
The ROC analysis showed that the MPV and MPR could discriminate SLE from RA with reasonable sensitivity and specificity in our study. The MPRs of patients with rheumatic inflammatory diseases were similar to those of the HC group but were higher in the SLE group than in the RA group in our study. This finding could be useful in the differential diagnosis of SLE and RA. The MPV is associated with atherosclerotic conditions, such as hypertension, diabetes mellitus, smoking, hyperlipidemia, and myocardial infarction $[22,25]$. The MPV values were slightly higher in the BD and SLE groups than in the other groups. When the vascular nature of these diseases is considered, it can be concluded that the MPV value is elevated in chronic inflammatory diseases with coexisting vascular pathology. Previous studies have to be evaluated for this conclusion.

The PLR was significantly higher only in the SLE group among studied rheumatic inflammatory diseases in our study. This finding suggests that the PLR may be an important biomarker for the diagnosis of SLE.

The NLR was significantly higher in the RA, SSc, and SLE groups. However, it was not increased in the BD group in our study. These findings could indicate that a high NLR might be used as a biomarker for the presence of the collagen tissue diseases. Similarly, the NLR was previously reported to be increased in patients with RA [3]. On the other hand, the level of NLR was documented to be altered in AS, which is an inflammatory disease [3], chronic kidney disease [26], and atherosclerotic vascular diseases [27]. The cause of an increased NLR level is increased cytokines and the inflammatory process $[3,26,28]$. 
The NLR was significantly higher in hypocomplementemic than in normocomplementemic SLE patients. Decreased complement is accepted as an indicator for disease activity and lupus nephritis. Thus, the NLR could be an indicator of disease activity and lupus nephritis. However, there was no significant difference in the NLR of active and inactive SLE patients or in the NLR of antids-DNA-positive and negative patients in our study. Qin et al. [5] reported that NLR was associated with disease activity and the presence of nephritis. Conversely, Oehadian et al. [11] demonstrated that it was not related with disease activity and clinical subsets.

The NLR levels were similar in the BD and HC groups in our study. Conversely, previous studies $[9,23]$ showed higher NLR levels in patients with BD than in healthy subjects. On the other hand, it was significantly higher in the active $\mathrm{BD}$ patients than in the inactive $\mathrm{BD}$ cases in our study. It was also significantly higher in the patients with neuro-BD than in those without neuro-BD, and higher in patients with active genital ulcers than those without. In addition, there was a positive correlation between the NLR and the ESR and BSAS in the BD group. These data show that the NLR may be an important bio-index for detecting $\mathrm{BD}$ activity and the presence of neuro-BD. On the other hand, the NLR was elevated in all inflammatory diseases, but not in quiescent $\mathrm{BD}$. This finding could be attributed to the nature of $\mathrm{BD}$, which is characterized by a recurrent pattern of presentation, with periods of remission and flare-ups.
The limitations of this study were that a power analysis was not performed to detect the numbers of enrolled participants in the study, as well as its cross-sectional design.

\section{Conclusion}

The MPV and MPR were significantly higher in the SLE group than in the RA group in our study. The PLR was also significantly higher in the SLE group than in the HC group. These results suggest that the MPV, MPR, and PLR are useful diagnostic tools for SLE. On the other hand, the NLR was significantly higher in the hypocomplementemic patients than in the normocomplementemic patients in the SLE group. It was also higher in the active $\mathrm{BD}$ patients than in inactive $\mathrm{BD}$ patients, and was significantly higher in the patients with neuro-BD than in those without. The NLR can be useful to detect the disease activities of SLE and BD. However, further studies that include more patients and subgroups are needed for more precise elucidation and clinical usage of these indices.

\section{Disclosure Statement}

The authors have no conflicts of interest to declare.

\section{References}

1 Kisacik B, Tufan A, Kalyoncu U, et al: Mean platelet volume (MPV) as an inflammatory marker in ankylosing spondylitis and rheumatoid arthritis. Joint Bone Spine 2008;75: 291-294.

12 Yazici S, Yazici M, Erer B, et al: The platelet indices in patients with rheumatoid arthritis: mean platelet volume reflects disease activity. Platelets 2010;21:122-125.

-3 Mercan R, Bitik B, Tufan A, et al: The association between neutrophil/lymphocyte ratio and disease activity in rheumatoid arthritis and ankylosing spondylitis. J Clin Lab Anal 2015, DOI: 10.1002/jcla.21908.

4 Safak S, Uslu AU, Serdal K, et al: Association between mean platelet volume levels and inflammation in SLE patients presented with arthritis. Afr Health Sci 2014;14:919-924.
5 Qin B, Ma N, Tang Q, et al: Neutrophil to lymphocyte ratio (NLR) and platelet to lymphocyte ratio (PLR) were useful markers in assessment of inflammatory response and disease activity in SLE patients. Mod Rheumatol 2016;26:372-376.

6 Soydinc S, Turkbeyler IH, Pehlivan Y, et al: Mean platelet volume seems to be a valuable marker in patients with systemic sclerosis. Inflammation 2014;37:100-106.

7 Canpolat F, Akpınar H, Eskioğlu F: Mean platelet volume in psoriasis and psoriatic arthritis. Clin Rheumatol 2010;29:325-328.

8 Acikgoz N, Karincaoglu Y, Ermis N, et al: Increased mean platelet volume in Behçet's disease with thrombotic tendency. Tohoku J Exp Med 2010;221:119-123.

-9 Rifaioglu EN, Bülbül Şen B, Ekiz Ö, et al: Neutrophil to lymphocyte ratio in Behçet's disease as a marker of disease activity. Acta Dermatovenerol Alp Pannonica Adriat 2014; 23:65-67.
10 Azab B, Torbey E, Singh J, et al: Mean platelet volume/platelet count ratio as a predictor of long-term mortality after non-ST-elevation myocardial infarction. Platelets 2011;22:557566.

11 Oehadian A, Suryadinata H, Dewi S, et al: The role of neutrophyl lymphocyte count ratio as an inflammatory marker in systemic lupus erythematosus. Acta Med Indones 2013;45: 170-174.

12 Yazici S, Yazici M, Erer B, et al: The platelet functions in patients with ankylosing spondylitis: anti-TNF- $\alpha$ therapy decreases the mean platelet volume and platelet mass. Platelets 2010;21:126-131.

$\checkmark 13$ Hochberg MC: Updating the American College of Rheumatology revised criteria for the classification of systemic lupus erythematosus. Arthritis Rheum 1997;40:1725.

14 Masi AT: Preliminary criteria for the classification of systemic sclerosis (scleroderma). Arthritis Rheum 1980;23:581-590. 
15 Weichsler B, Davatchi F, Mizushima Y, et al Criteria for diagnosis of Behçet's disease. Lancet 1990;335:1078-1080.

16 Aletaha D, Neogi T, Silman AJ, et al: 2010 rheumatoid arthritis classification criteria: an American College of Rheumatology/European League against Rheumatism collaborative initiative. Ann Rheum Dis 2010;69:1580-1588.

17 Medsger T Jr, Silman A, Steen V, et al: A disease severity scale for systemic sclerosis: development and testing. J Rheumatol 1999;26: 2159-2167.

18 Bombardier C, Gladman DD, Urowitz MB, et al: Derivation of the SLEDAI: a disease activity index for lupus patients. Arthritis Rheum 1992;35:630-640.

19 Gladman D, Ginzler E, Goldsmith C, et al: The development and initial validation of the Systemic Lupus International Collaborating Clinics/American College of Rheumatology damage index for systemic lupus erythematosus. Arthritis Rheum 1996;39:363-369.
20 Yilmaz S, Simsek I, Cinar M, et al: Patientdriven assessment of disease activity in Behçet's syndrome: cross-cultural adaptation, reliability and validity of the Turkish version of the Behçet's Syndrome Activity Score. Clin Exp Rheumatol 2013;31:77-83.

21 Aletaha D, Smolen J: The Simplified Disease Activity Index (SDAI) and the Clinical Disease Activity Index (CDAI): a review of their usefulness and validity in rheumatoid arthritis. Clin Exp Rheumatol 2005; 23:S100.

22 Gasparyan AY, Stavropoulos-Kalinoglou A, Toms TE, et al: Association of mean platelet volume with hypertension in rheumatoid arthritis. Inflamm Allergy Drug Targets 2010;9: 45-50.

23 Ozturk C, Balta S, Balta I, et al: Neutrophillymphocyte ratio and carotid-intima media thickness in patients with Behçet disease without cardiovascular involvement. Angiology 2015;66:291-296.
24 Lancé MD, van Oerle R, Henskens YM, et al: Do we need time adjusted mean platelet volume measurements? Lab Hematol 2010;16: 28-31.

25 Kodiatte TA, Manikyam UK, Rao SB, et al: Mean platelet volume in type 2 diabetes mellitus. J Lab Physicians 2012;4:5.

26 Okyay GU, Inal S, Onec K, et al: Neutrophil to lymphocyte ratio in evaluation of inflammation in patients with chronic kidney disease. Ren Fail 2013;35:29-36.

27 Nacar AB, Erayman A, Kurt M, et al: The relationship between coronary collateral circulation and neutrophil/lymphocyte ratio in patients with coronary chronic total occlusion. Med Princ Pract 2015;24:65-69.

28 Ahsen A, Ulu MS, Yuksel S, et al: As a new inflammatory marker for familial Mediterranean fever: neutrophil-to-lymphocyte ratio. Inflammation 2013;36:1357-1362. 